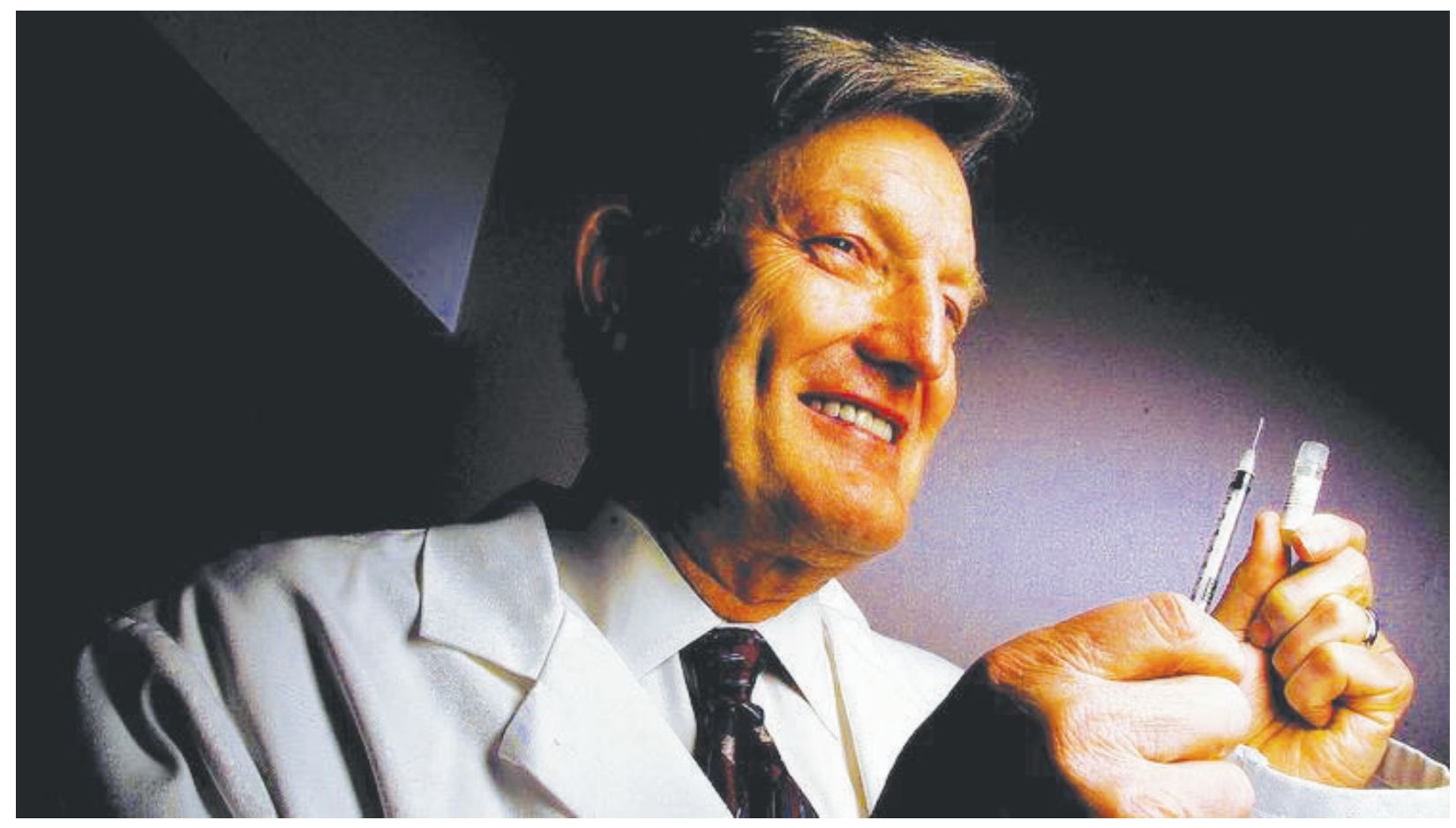

\title{
Donald L. Morton, MD 1934-2014
}

Donald Lee Morton was born on September 12, 1934 in rural West Virginia. After his graduation from the University of California-Berkeley, he went on to receive his medical degree from the University of California-San Francisco. In 1969, he was appointed head of the tumor immunology section and senior surgeon at the surgery branch of the National Cancer Institute at the National Institutes of Health and soon became recognized as one of the early pioneers of translational clinical cancer immunology research. Dr. Morton was a natural-born leader, as reflected through his many successful leadership positions throughout his life. In 1971, he became professor and chief of surgical oncology at University of California-Los Angeles and was promoted to chief of the Department of General Surgery in 1977. He became a professor emeritus of surgery in 1991. Dr. Morton served as president of the Society of Surgical Oncology, president of the World Federation of Surgical Oncology Societies, and founding president of the International Sentinel Node Society. He also mentored over 150 fellows in translational research and surgical treatment of cancer throughout his career. He was always eager to discuss both his clinical and translational research experience with all investigators, young and old.

Dr. Morton's early groundbreaking work in melanoma remains the foundation of the current surgical oncology procedures, immunotherapy treatment, and diagnostic approaches, not only for melanoma, but also for numerous other cancers. Among his many major contributions to surgical oncology is the development of the sentinel lymph node biopsy procedure for early-stage primary melanoma and other solid tumors. This procedure 
resulted in thousands of publications and is still practiced all over the world. Morton's cuttingedge work on cancer-targeting antibodies led to the development of adjuvant therapies and melanoma vaccine phase II/III clinical trials. His academic leadership led to the development of four major international multicenter clinical trials in melanoma. The Multicenter Selective Lymphadenectomy Trial (MSLT-II) involves over 60 centers worldwide and is now completed and in the follow-up stage. During his career, Dr. Morton received many awards in surgery, including the Jacobson Innovation Award from American College of Surgeons.
Among his countless legacies, Morton founded the John Wayne Cancer Institute in Santa Monica, California. Morton's leadership positions within JWCI include acting as its president, board member, medical director, chief of the melanoma program, director of the surgical oncology fellowship program, and distinguished professor of surgery. He established the surgical oncology fellowship program at the University of California-Los Angeles Department of Surgery in the 1970s and brought it to the JWCI in 1991. The program is still active at JWCI. JWCI's exceptional melanoma biospecimen repository is yet another legacy that Dr. Donald Morton left behind. 